\title{
Optical Absorption in Twisted Bilayer Graphene
}

\author{
Pilkyung Moon and Mikito Koshino \\ Department of Physics, Tohoku University, Sendai, 980-8578, Japan
}

(Dated: February 26, 2013)

\begin{abstract}
We theoretically study the optical absorption property of twisted bilayer graphenes with various stacking geometries, and demonstrate that the spectroscopic characteristics serve as a fingerprint to identify the rotation angle between two layers. We find that the absorption spectrum almost continuously evolves in changing the rotation angle, regardless of the lattice commensurability. The spectrum is characterized by series of peaks associated with the van Hove singularity, and the peak energies systematically shift with the rotation angle. We calculate the optical absorption in two different frameworks; the tight-binding model and the effective continuum model based on the Dirac equation. For small rotation angles less than $10^{\circ}$, the effective model well reproduces the lowenergy band structure and the optical conductivity of the tight-binding model, and also explains the optical selection rule analytically in terms of the symmetry of the effective Hamiltonian.
\end{abstract}

\section{INTRODUCTION}

The recent advances in fabrication of atomically-thin materials ${ }^{1-5}$ have realized a new kind of two-dimensional superlattice in which the lattice mismatch between neigh-
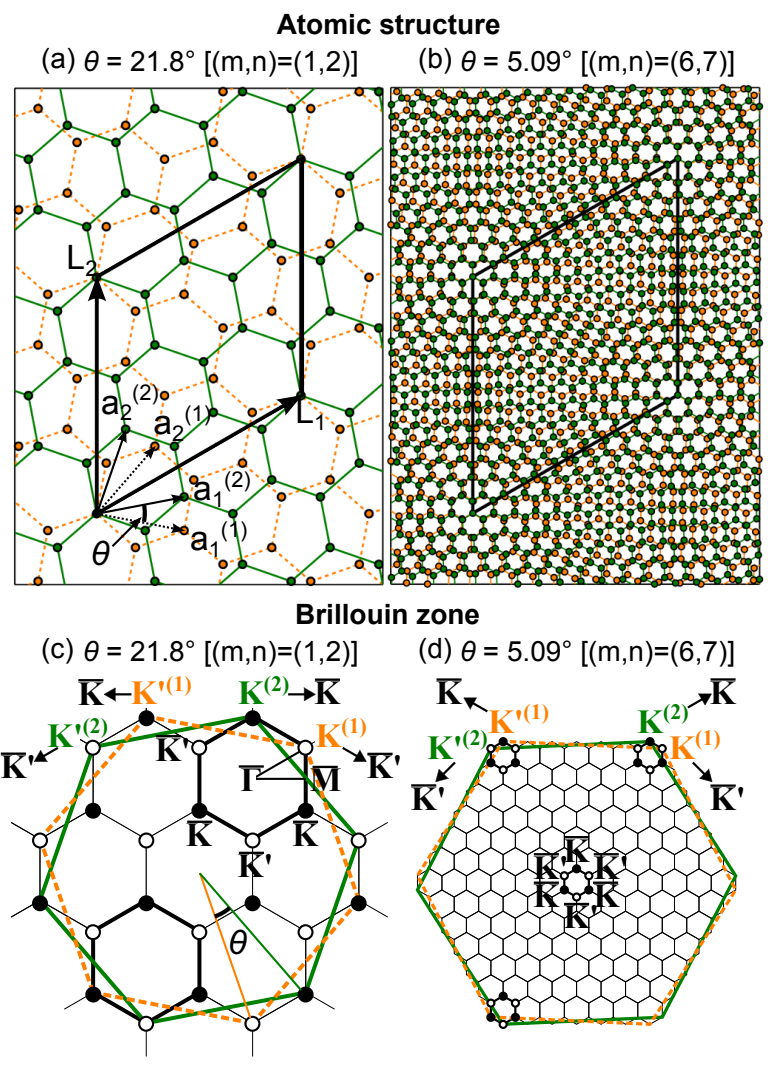

FIG. 1: Atomic structures of TBGs with (a) $\theta=21.8^{\circ}$ and (b) $\theta=5.09^{\circ}$. Dashed (orange) and solid (green) lines represent the lattices of layers 1 and 2, respectively. Brillouin zone of TBGs with (c) $\theta=21.8^{\circ}$ and (d) $\theta=5.09^{\circ}$. Dashed (orange) and solid (green) large hexagons indicate the first Brillouin zone of layer 1 and 2, respectively, and thick small-hexagon is the folded Brillouin zone of TBG. boring layers gives rise to an additional potential modulation. One example of such systems is twisted bilayer graphene (TBG), in which two graphene layers are stacked with an arbitrary orientation. 1,6-10 In TBG, the interlayer interaction between two misoriented layers significantly modifies the low-energy band structure, arising novel electronic features distinct from intrinsic graphene. In decreasing the rotation angle, the interference between two lattice periods produces a Moiré pattern with a long wavelength, where the characteristic features such as band gaps and van Hove singularity appear in the farinfrared region, and the band velocity of Dirac cone is significantly reduced. 2,7,11-16 Recently, the band properties of TBG has been probed by Raman spectroscopy, $, 2,17-19$ optical reflection spectroscopy $\stackrel{20}{2}$ angle-resolved photoemission spectroscopy, 21 and by terahertz time-domain spectroscopy. ${ }^{22}$

The purpose of this paper is to reveal the optical absorption properties of TBGs with various stacking geometries. The optical absorption measurement is widely adopted for graphene-based systems to investigate the electronic structures ${ }^{23}-34$ Theoretically, the optical absorption for light incident perpendicular to the layer is related to the dynamical conductivity, which was calculated for monolayer graphene, ${ }^{35-38} A B$-stacked graphene bilayer and multilayers, $39-44$ and also for TBG with a specific rotation angle. 20,45 In this paper, we calculate the dynamical conductivity of TBG in a wide range of rotation angles, and demonstrate that the spectroscopic characteristics serve as a fingerprint to identify the stacking geometry. We find that the spectrum is characterized by series of peaks associated with the van Hove singularity, of which transition energy continuously shifts as the rotation angle is changed. Here the dynamical conductivity is calculated in two different frameworks; the tight-binding model and the effective continuum model based on the Dirac equation. For the latter, we develop a general treatment to derive the effective model from arbitrary tight-binding parametrization. For small rotation angles less than $10^{\circ}$, the effective model nicely reproduces the low-energy band structure and the dynamical conductivity of the tight-binding model, and also explains the 
(a) $\theta=13.2^{\circ}[(m, n)=(2,3)]$

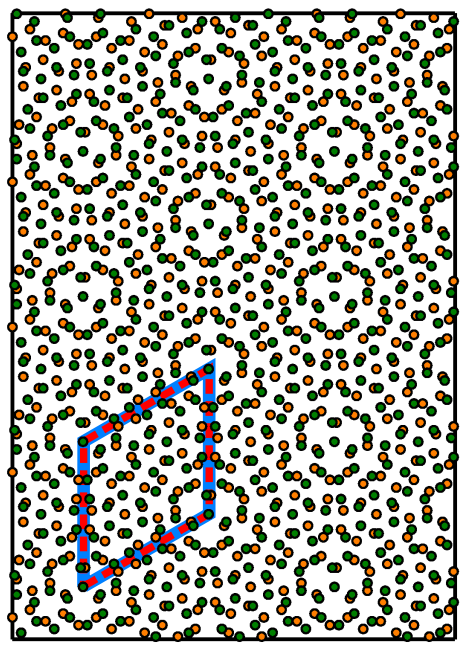

(b) $\theta=11.0^{\circ}[(\mathrm{m}, \mathrm{n})=(5,7)]$

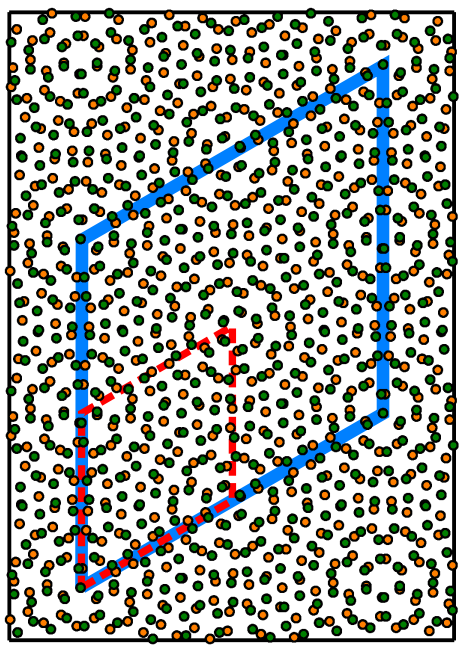

(c) $\theta=9.43^{\circ}[(m, n)=(3,4)]$

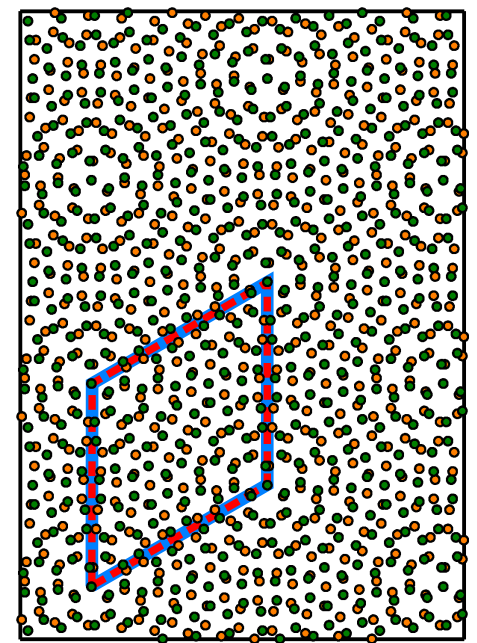

: Moiré unit cell

$\checkmark$ : Rigorous superlattice unit cell

FIG. 2: Atomic structures of TBGs with (a) $\theta=13.2^{\circ}$, (b) $11.0^{\circ}$, and (c) $9.43^{\circ}$. Dashed (red) and solid (blue) parallelograms correspond to the Moiré unit cell and rigorous superlattice unit cell, respectively.

optical selection rule analytically in terms of the symmetry of the effective Hamiltonian.

\section{THEORETICAL METHODS}

\section{A. Atomic structure and Brillouin zone}

Graphene is a single layer of carbon atoms arranged in a honeycomb lattice structure, of which unit cell includes two inequivalent sublattice sites, $A$ and $B$. The stacking geometry of bilayer graphene is characterized by the relative rotation angle $\theta$ combined with the lateral translation $\boldsymbol{\delta}$ between the layers. Here we define the structure of TBG by rotating the layer 1 and 2 of $A A$-stacked bilayer around a common $B$-site by $-\theta / 2$ and $+\theta / 2$, respectively, and then translating the layer 2 relatively to the layer 1 by $\boldsymbol{\delta}$. We define $\mathbf{a}_{1}=a(1,0)$ and $\mathbf{a}_{2}=a(1 / 2, \sqrt{3} / 2)$ as the lattice vectors of the $A A$-stacked bilayer before the rotation, where $a \approx 0.246 \mathrm{~nm}$ is the lattice constant. The lattice vectors of the layer $l$ after the rotation are given by $\mathbf{a}_{i}^{(l)}=R(\mp \theta / 2) \mathbf{a}_{i}$ with $\mp$ for $l=1,2$, respectively, where $R(\theta)$ represents the rotation by $\theta$.

When $\boldsymbol{\delta}$ is fixed to 0 , the rotation $\theta=0$ and $60^{\circ}$ give $A A$ and $A B$ stacking, respectively. $60^{\circ}-\theta$ is equivalent to $-\theta$ followed by a relative translation of the layer 2 from $A$ site to $B$ site. $\underline{\underline{46} . \underline{47}}$ Also, $\theta$ and $-\theta$ are mirror images sharing equivalent band structures. Therefore, it is reasonable to characterize the geometry of TBG by the combination of $\theta\left(0 \leq \theta \leq 30^{\circ}\right)$ and $\boldsymbol{\delta}$.

The lattice structure of TBG is not periodic in general angles because the periods of two graphene layers are generally incommensurate with each layer. But in some special angles where two periods happen to match, the structure becomes rigorously periodic giving a finite unit cell. This takes place when $\theta$ coincides with the angle between $\mathbf{v}_{1}=m \mathbf{a}_{1}+n \mathbf{a}_{2}$ and $\mathbf{v}_{2}=n \mathbf{a}_{1}+m \mathbf{a}_{2}$ with certain integers $m$ and $n$, because then the lattice points $\mathbf{v}_{1}$ on the layer 1 and $\mathbf{v}_{2}$ on the layer 2 of the non-rotated bilayer graphene merge after the rotation $\theta / 2$ and $-\theta / 2$, respectively. The lattice vectors of the superlattice unit cell are thus given by$\underline{46}$

$$
\mathbf{L}_{1}=m \mathbf{a}_{1}^{(1)}+n \mathbf{a}_{2}^{(1)}=n \mathbf{a}_{1}^{(2)}+m \mathbf{a}_{2}^{(2)}
$$

and $\mathbf{L}_{2}=R(\pi / 3) \mathbf{L}_{1}$. The rotation angle $\theta$ is related to $(m, n)$ by

$$
\cos \theta=\frac{1}{2} \frac{m^{2}+n^{2}+4 m n}{m^{2}+n^{2}+m n} .
$$

The lattice constant $L=\left|\mathbf{L}_{1}\right|=\left|\mathbf{L}_{2}\right|$ is

$$
L=a \sqrt{m^{2}+n^{2}+m n}=\frac{|m-n| a}{2 \sin (\theta / 2)} .
$$

Figures 1(a) and 1(b) show the atomic structures of TBG with two different rotation angles $\theta=21.8^{\circ}$ $[(m, n)=(1,2)]$ and $5.09^{\circ}[(m, n)=(6,7)]$, respectively. Unless otherwise noted, $\boldsymbol{\delta}=0$ is used. Figures 1(c) and 1(d) show the corresponding Brillouin zone in the extended scheme. In each figure, dashed (orange) and solid (green) large hexagons correspond to the first Brillouin zones of layer 1 and 2, respectively, and thick small hexagon to the folded Brillouin zone of TBG. $K^{(l)}$ and $K^{\prime(l)}$ denote the two inequivalent valleys of layer $l$. The four valleys $K^{(1)}, K^{\prime(1)}, K^{(2)}$, and $K^{\prime(2)}$ of the two layers are folded back to the two Dirac points, $\bar{K}$ and $\bar{K}^{\prime}$, in the folded Brillouin zone $\underline{\underline{13}}$ 
When the rotation angle is small, the mismatch between the lattice vectors of the two layers gives rise to a Moiré pattern with a long spatial period as seen in Fig. [1(b) $\stackrel{13,48}{=}$ The local lattice structure near a certain point $\mathbf{r}$ approximates a non-rotated bilayer graphene with displacement $\boldsymbol{\delta}$, which depends on the position as

$$
\boldsymbol{\delta}(\mathbf{r})=2 \sin (\theta / 2)\left(\mathbf{e}_{z} \times \mathbf{r}\right),
$$

where $\mathbf{r}$ is measured from the center of the rotation, and $\mathbf{e}_{z}$ is a unit vector perpendicular to the plane. The period of the Moiré pattern $\mathbf{L}_{i}^{\mathrm{M}}$ can be obtained by the condition that $\boldsymbol{\delta}\left(\mathbf{L}_{i}^{\mathrm{M}}\right)$ coincides with a primitive lattice vector of the original $A A$-stacked bilayer. We may choose

$$
\mathbf{L}_{1}^{\mathrm{M}}=\frac{\left(-\mathbf{a}_{1}+\mathbf{a}_{2}\right) \times \mathbf{e}_{z}}{2 \sin (\theta / 2)}, \quad \mathbf{L}_{2}^{\mathrm{M}}=\frac{-\mathbf{a}_{1} \times \mathbf{e}_{z}}{2 \sin (\theta / 2)},
$$

giving $\boldsymbol{\delta}\left(\mathbf{L}_{1}^{\mathrm{M}}\right)=-\mathbf{a}_{1}+\mathbf{a}_{2}$ and $\boldsymbol{\delta}\left(\mathbf{L}_{2}^{\mathrm{M}}\right)=-\mathbf{a}_{1}$. The lattice constant $L_{\mathrm{M}}=\left|\mathbf{L}_{1}^{\mathrm{M}}\right|=\left|\mathbf{L}_{2}^{\mathrm{M}}\right|$ is

$$
L_{\mathrm{M}}=\frac{a}{2 \sin (\theta / 2)} \text {. }
$$

The Moiré superlattice vectors $\mathbf{L}_{i}^{\mathrm{M}}$ can be always defined for any $\theta$, even when the lattice structure is incommensurate. At commensurate angles, the rigorous superlattice period $L$ is exactly $|m-n|$ times bigger than the Moiré period $L_{\mathrm{M}}$. In Fig. 2, we illustrate the lattice structures of $\theta=13.2^{\circ}[(m, n)=(2,3)], \theta=11.0^{\circ}$ $[(m, n)=(5,7)]$ and $\theta=9.43^{\circ}[(m, n)=(3,4)]$. We can see that the atomic structure exactly matches the Moiré pattern in $13.2^{\circ}$ and $9.43^{\circ}(|m-n|=1)$, while in $11.0^{\circ}$, the exact period $L$ is twice as large as $L_{\mathrm{M}}$ since $|m-n|=2$, and accordingly the atomic structure is slightly different between neighboring units in the Moiré pattern.

\section{B. Tight-binding model}

We calculate the eigenenergies and eigenfunctions in TBG using the tight-binding model for $p_{z}$ atomic orbitals. The Hamiltonian is written as

$$
H=-\sum_{\langle i, j\rangle} t\left(\mathbf{R}_{i}-\mathbf{R}_{j}\right)\left|\mathbf{R}_{i}\right\rangle\left\langle\mathbf{R}_{j}\right|+\text { H.c. },
$$

where $\mathbf{R}_{i}$ and $\left|\mathbf{R}_{i}\right\rangle$ represent the lattice point and the atomic state at site $i$, respectively, and $t\left(\mathbf{R}_{i}-\mathbf{R}_{j}\right)$ is the transfer integral between the sites $i$ and $j$. We adopt an approximation, $14.49-51$

$$
\begin{aligned}
& -t(\mathbf{d})=V_{p p \pi}\left[1-\left(\frac{\mathbf{d} \cdot \mathbf{e}_{z}}{d}\right)^{2}\right]+V_{p p \sigma}\left(\frac{\mathbf{d} \cdot \mathbf{e}_{z}}{d}\right)^{2} \\
& V_{p p \pi}=V_{p p \pi}^{0} \exp \left(-\frac{d-a_{0}}{\delta_{0}}\right) \\
& V_{p p \sigma}=V_{p p \sigma}^{0} \exp \left(-\frac{d-d_{0}}{\delta_{0}}\right)
\end{aligned}
$$

where $a_{0}=a / \sqrt{3} \approx 0.142 \mathrm{~nm}$ is the distance of neighboring $A$ and $B$ sites on monolayer, and $d_{0} \approx 0.335 \mathrm{~nm}$ is the interlayer spacing. $V_{p p \pi}^{0}$ is the transfer integral between the nearest-neighbor atoms of monolayer graphene and $V_{p p \sigma}^{0}$ is that between vertically located atoms on the neighboring layers. Here we take $V_{p p \pi}^{0} \approx-2.7 \mathrm{eV}$, $V_{p p \sigma}^{0} \approx 0.48 \mathrm{eV}$, to fit the dispersions of monolayer graphene and $A B$-stacked bilayer graphene. $\underline{\underline{14}} \delta_{0}$ is the decay length of the transfer integral, and is chosen as $0.184 a$ so that the next nearest intralayer coupling becomes $0.1 V_{p p \pi}^{0} \stackrel{14,50}{\underline{s}}$ The transfer integral for $d>4 a_{0}$ is exponentially small and can be safely neglected.

\section{Effective continuum model}

When the rotation angle is small and the Moiré superlattice period is much larger than the lattice constant, the interaction between the two graphene layers has only the long-wavelength components, allowing one to treat the problem in the effective continuum model. The continuum approaches for TBG have been introduced in several literatures $11,15,16,52$ Here we develop a general treatment to construct an effective model directly from the tightbinding Hamiltonian in Eq. (7). To construct the Hamiltonian matrix, we define the Bloch wave basis of a single layer as

$$
\begin{aligned}
& \left|\mathbf{k}, A_{l}\right\rangle=\frac{1}{\sqrt{N}} \sum_{\mathbf{R}_{A_{l}}} e^{i \mathbf{k} \cdot \mathbf{R}_{A_{l}}}\left|\mathbf{R}_{A_{l}}\right\rangle, \\
& \left|\mathbf{k}, B_{l}\right\rangle=\frac{1}{\sqrt{N}} \sum_{\mathbf{R}_{B_{l}}} e^{i \mathbf{k} \cdot \mathbf{R}_{B_{l}}}\left|\mathbf{R}_{B_{l}}\right\rangle,
\end{aligned}
$$

where the position $\mathbf{R}_{A_{l}}\left(\mathbf{R}_{B_{l}}\right)$ runs over all $A(B)$ sites on the layer $l(=1,2), N$ is the number of monolayer's unit cell in the whole system, and $\mathbf{k}$ is two-dimensional Bloch wave vector defined in the first Brillouin zone of monolayer on the layer $l$.

Intralayer matrix element of each layer occurs only within the same wave vector, and it is given by

$$
\begin{aligned}
h_{A_{l} A_{l}}(\mathbf{k}) & \equiv\left\langle\mathbf{k}, A_{l}|H| \mathbf{k}, A_{l}\right\rangle=h(\mathbf{k}, 0), \\
h_{A_{l} B_{l}}(\mathbf{k}) & \equiv\left\langle\mathbf{k}, A_{l}|H| \mathbf{k}, B_{l}\right\rangle=h\left(\mathbf{k}, \boldsymbol{\tau}_{1}\right), \\
h_{B_{l} B_{l}}(\mathbf{k}) & =h_{A_{l} A_{l}}(\mathbf{k}),
\end{aligned}
$$

where $\boldsymbol{\tau}_{1}=\left(2 \mathbf{a}_{2}-\mathbf{a}_{1}\right) / 3$ is a vector connecting from $\mathrm{B}$ site to A site, and

$$
\begin{aligned}
h(\mathbf{k}, \boldsymbol{\tau})=\sum_{n_{1}, n_{2}} & -t\left(n_{1} \mathbf{a}_{1}+n_{2} \mathbf{a}_{2}+\boldsymbol{\tau}\right) \\
& \times \exp \left[i \mathbf{k} \cdot\left(n_{1} \mathbf{a}_{1}+n_{2} \mathbf{a}_{2}+\boldsymbol{\tau}\right)\right] .
\end{aligned}
$$

The low-energy spectrum of the monolayer graphene is approximated by effective Dirac cones centered at $K$ and $K^{\prime}$ points $\stackrel{53-57}{-5 e}$ take $\mathbf{K}=(2 \pi / a)(-2 / 3,0)$ and $\mathbf{K}^{\prime}=$ $(2 \pi / a)(2 / 3,0)$ as the $K$-points of non-rotated graphene. The $K$ points of the layer $l$ are then given by $\mathbf{K}^{(l)}=$ 
$R(\mp \theta / 2) \mathbf{K}$ and $\mathbf{K}^{\prime(l)}=R(\mp \theta / 2) \mathbf{K}^{\prime}$, with $\mp$ for $l=1$ and 2 , respectively. When $\mathbf{k}$ is close to either of $K$ or $K^{\prime}$ the intralayer matrix element is approximately written as ${ }^{57}$,

$$
\begin{aligned}
& h_{A_{l} B_{l}}(\mathbf{k}) \\
& \approx \begin{cases}-\hbar v\left[\left(k_{x}-K_{x}^{(l)}\right)-i\left(k_{y}-K_{y}^{(l)}\right)\right] e^{-i \eta^{(l)}} & (\mathbf{k} \approx \mathbf{K}), \\
-\hbar v\left[-\left(k_{x}-K_{x}^{\prime(l)}\right)-i\left(k_{y}-K_{y}^{\prime(l)}\right)\right] e^{i \eta^{(l)}} & \left(\mathbf{k} \approx \mathbf{K}^{\prime}\right),\end{cases}
\end{aligned}
$$

where $\eta^{(l)}= \pm \theta / 2$ for $l=1$ and 2 , respectively. In the following we neglect the phase factor $e^{-i \eta^{(l)}}$ assuming $\theta \ll 1$. The parameter $v$ is the band velocity of the Dirac cone, which is given in the present tight-binding parametrization as

$$
v \approx \frac{\sqrt{3}}{2} \frac{a}{\hbar} V_{p p \pi}^{0}\left(1-2 e^{-a_{0} / \delta_{0}}\right),
$$

where the first and the second terms in the bracket originate from the hopping between the first and the second nearest $A B$ pairs, respectively. The diagonal matrix element $h_{A_{l} A_{l}}(\mathbf{k})=h_{B_{l} B_{l}}(\mathbf{k})$ are shown to be of the order of $|\mathbf{k}-\mathbf{K}|^{2}$ and $\left|\mathbf{k}-\mathbf{K}^{\prime}\right|^{2}$ near $K$ and $K^{\prime}$ points, respectively, and will be neglected in the following.

For the interlayer coupling, we first consider a nonrotated bilayer graphene with $\theta=0$ and a fixed lattice displacement $\boldsymbol{\delta}$. The unit cell is spanned by monolayer's lattice vectors, $\mathbf{a}_{1}$ and $\mathbf{a}_{2}$, which are now shared by both layers. As the system has the same periodicity as the monolayer, the interlayer coupling occurs within states belonging to the same $\mathbf{k}$. The interlayer matrix element is written as

$$
\begin{aligned}
U_{A_{2} A_{1}}(\mathbf{k}, \boldsymbol{\delta}) & \equiv\left\langle\mathbf{k}, A_{2}|H| \mathbf{k}, A_{1}\right\rangle=u(\mathbf{k}, \boldsymbol{\delta}) \\
U_{B_{2} B_{1}}(\mathbf{k}, \boldsymbol{\delta}) & \equiv\left\langle\mathbf{k}, B_{2}|H| \mathbf{k}, B_{1}\right\rangle=u(\mathbf{k}, \boldsymbol{\delta}) \\
U_{B_{2} A_{1}}(\mathbf{k}, \boldsymbol{\delta}) & \equiv\left\langle\mathbf{k}, B_{2}|H| \mathbf{k}, A_{1}\right\rangle=u\left(\mathbf{k}, \boldsymbol{\delta}-\boldsymbol{\tau}_{1}\right) \\
U_{A_{2} B_{1}}(\mathbf{k}, \boldsymbol{\delta}) & \equiv\left\langle\mathbf{k}, A_{2}|H| \mathbf{k}, B_{1}\right\rangle=u\left(\mathbf{k}, \boldsymbol{\delta}+\boldsymbol{\tau}_{1}\right),(14)
\end{aligned}
$$

where

$$
\begin{aligned}
u(\mathbf{k}, \boldsymbol{\delta})= & \sum_{n_{1}, n_{2}}-t\left(n_{1} \mathbf{a}_{1}+n_{2} \mathbf{a}_{2}+d_{0} \mathbf{e}_{z}+\boldsymbol{\delta}\right) \\
& \times \exp \left[-i \mathbf{k} \cdot\left(n_{1} \mathbf{a}_{1}+n_{2} \mathbf{a}_{2}+\boldsymbol{\delta}\right)\right] .
\end{aligned}
$$

$u(\mathbf{k}, \boldsymbol{\delta})$ can be immediately calculated by taking a summation over some small $n_{i}$ 's, since $t(\mathbf{d})$ rapidly vanishes in $|\mathbf{d}| \gg a$.

When $\theta$ is slightly shifted from zero to a small finite angle, the local lattice structure is approximately viewed as a non-rotated bilayer graphene, where the displacement $\boldsymbol{\delta}$ slowly depends on the position $\mathbf{r}$ in accordance with Eq. (4). Then the interlayer interaction couples wave vectors $\mathbf{k}$ and $\mathbf{k}^{\prime}$ which are close to each other such that $\left|\mathbf{k}^{\prime}-\mathbf{k}\right| \ll 2 \pi / a$. The interlayer matrix element is approximately written as

$$
\begin{aligned}
& \left\langle\mathbf{k}^{\prime}, X_{2}^{\prime}|H| \mathbf{k}, X_{1}\right\rangle \approx \\
& \frac{1}{\Omega_{\mathrm{M}}} \int_{\Omega_{\mathrm{M}}} d \mathbf{r} U_{X_{2}^{\prime} X_{1}}\left[\frac{\mathbf{k}+\mathbf{k}^{\prime}}{2}, \boldsymbol{\delta}(\mathbf{r})\right] e^{-i\left(\mathbf{k}^{\prime}-\mathbf{k}\right) \cdot \mathbf{r}},
\end{aligned}
$$

where $X$ and $X^{\prime}$ are either of $A$ or $B, U_{X_{2}^{\prime}, X_{1}}$ are the interlayer coupling in non-rotational bilayer in Eq. (14), and $\Omega_{\mathrm{M}}=\left|\mathbf{L}_{1}^{\mathrm{M}} \times \mathbf{L}_{2}^{\mathrm{M}}\right|$ is the Moiré superlattice unit cell. The derivation of Eq. (16) is detailed in Appendix A. $U_{X_{2}^{\prime} X_{1}}[\mathbf{q}, \boldsymbol{\delta}(\mathbf{r})]$ is periodic in $\mathbf{r}$ with the Moiré superlattice periods, and therefore the matrix element Eq. (16) is nonzero only when $\mathbf{k}^{\prime}-\mathbf{k}=n_{1} \mathbf{G}_{1}^{\mathrm{M}}+n_{2} \mathbf{G}_{2}^{\mathrm{M}}$ where $\mathbf{G}_{i}^{\mathrm{M}}$ is the reciprocal lattice vector satisfying $\mathbf{L}_{i}^{\mathrm{M}} \cdot \mathbf{G}_{j}^{\mathrm{M}}=2 \pi \delta_{i j}$, and $n_{i}$ is an integer.

In TBG, the low-energy physics is still dominated by the states near $K$ and $K^{\prime}$ points because the interlayer coupling is much smaller than intralayer coupling. Besides, the states near $K$ and those near $K^{\prime}$ are far apart in the wave space when $\theta$ is small, so that they not hybridized by the interlayer coupling. Therefore, we may consider two valleys separately in constructing the Hamiltonian, and the factor $\left(\mathbf{k}+\mathbf{k}^{\prime}\right) / 2$ in Eq. (16) can be replaced with $\mathbf{K}$ or $\mathbf{K}^{\prime}$. In the real space representation, the effective Hamiltonian near $K$ is concisely written in the basis of $\left\{\left|A_{1}\right\rangle,\left|B_{1}\right\rangle,\left|A_{2}\right\rangle,\left|B_{2}\right\rangle\right\}$ as

$$
H_{\mathrm{eff}}=\left(\begin{array}{cc}
H_{1} & U^{\dagger} \\
U & H_{2}
\end{array}\right)
$$

with

$$
\begin{aligned}
& H_{l}=-\hbar v\left(\hat{\mathbf{k}}-\Delta \mathbf{K}^{(l)}\right) \cdot \boldsymbol{\sigma}, \\
& U=\left(\begin{array}{cc}
u(\mathbf{K}, \boldsymbol{\delta}) & u\left(\mathbf{K}, \boldsymbol{\delta}-\boldsymbol{\tau}_{1}\right) \\
u\left(\mathbf{K}, \boldsymbol{\delta}+\boldsymbol{\tau}_{1}\right) & u(\mathbf{K}, \boldsymbol{\delta})
\end{array}\right),
\end{aligned}
$$

where $\hat{\mathbf{k}}=-i \partial / \partial \mathbf{r}, \boldsymbol{\sigma}=\left(\sigma_{x}, \sigma_{y}\right)$ is the Pauli matrices, $\Delta \mathbf{K}^{(l)}=\mathbf{K}^{(l)}-\mathbf{K}$, and $\boldsymbol{\delta}=\boldsymbol{\delta}(\mathbf{r})$ is defined in Eq. (4). When deriving $H_{l}$ in Eq. (18) from Eq. (12), we replace $\mathbf{k}$ with $\hat{\mathbf{k}}+\mathbf{K}$, i.e., measure the wavenumber relatively to the common point $\mathbf{K}$ for both layers. The Hamiltonian for $K^{\prime}$ is obtained by replacing $\mathbf{K}$ with $\mathbf{K}^{\prime}$ and $\hat{k}_{x}$ with $-\hat{k}_{x}$ above. In the present choice of the tight-binding parameters $V_{p p \pi}^{0}, V_{p p \sigma}^{0}$ and $\delta_{0}$, the effective interlayer coupling $u(\mathbf{K}, \boldsymbol{\delta}(\mathbf{r}))$ is approximately written in terms of only a few Fourier components as

$u(\mathbf{K}, \boldsymbol{\delta}(\mathbf{r})) \approx(0.103 \mathrm{eV}) \times\left[1+e^{-i \mathbf{G}_{2}^{\mathrm{M}} \cdot \mathbf{r}}+e^{-i\left(\mathbf{G}_{1}^{\mathrm{M}}+\mathbf{G}_{2}^{\mathrm{M}}\right) \cdot \mathbf{r}}\right](1$

$u\left(\mathbf{K}^{\prime}, \boldsymbol{\delta}\right)$ is given by $u(\mathbf{K}, \boldsymbol{\delta})^{*}$. The expression of $u(\mathbf{K}, \boldsymbol{\delta})$ explicitly depends on the choice of $\mathbf{K}$ vector out of three equivalent corners in the Brillouin zone.

In the $k$-space representation, the Hamiltonian matrix can be written in the space of the single-layer bases at discrete $k$-points $\mathbf{k}=\mathbf{k}_{0}+n_{1} \mathbf{G}_{1}^{\mathrm{M}}+n_{2} \mathbf{G}_{2}^{\mathrm{M}}$, where $\mathbf{k}_{0}$ is a vector defined in the superlattice Brillouin zone spanned by $\mathbf{G}_{1}^{\mathrm{M}}$ and $\mathbf{G}_{2}^{\mathrm{M}} . \mathbf{k}_{0}=0$ corresponds to the $\bar{M}$ point. To obtain the energy spectrum and eigen wave function, we choose $k$-points satisfying $\hbar v|\mathbf{k}| \lesssim E_{\max }$ with a sufficiently large $E_{\max }$, and diagonalize the Hamiltonian within the limited wave space. To avoid a discrete change in the number of bases in varying $\mathbf{k}_{0}$, we adopt a soft cutoff which gradually reduces the matrix elements for the single-layer bases beyond $E_{\max }$. 
It is straightforward to show the Hamiltonian Eq. (17) has a certain symmetry expressed as

$$
\hat{\Sigma}^{-1} H_{\mathrm{eff}} \hat{\Sigma}=-H_{\mathrm{eff}}^{*},
$$

where

$$
\hat{\Sigma}=\left(\begin{array}{cc}
0 & \sigma_{x} \\
-\sigma_{x} & 0
\end{array}\right) .
$$

This immediately concludes that if $\psi$ is an eigenstate of $H_{\text {eff }}$ belonging to energy $E, \hat{\Sigma} \psi^{*}$ is an eigenstate of energy $-E$.

\section{Dynamical conductivity}

Using the eigen wave functions obtained by the tightbinding model or the effective continuum model, we calculate the dynamical conductivity

$$
\sigma_{x x}(\omega)=\frac{e^{2} \hbar}{i S} \sum_{\alpha, \beta} \frac{f\left(\varepsilon_{\alpha}\right)-f\left(\varepsilon_{\beta}\right)}{\varepsilon_{\alpha}-\varepsilon_{\beta}} \frac{\left|\left\langle\alpha\left|v_{x}\right| \beta\right\rangle\right|^{2}}{\varepsilon_{\alpha}-\varepsilon_{\beta}+\hbar \omega+i \eta}(22)
$$

where the sum is over all states, $S$ is the area of the system, $f(\varepsilon)$ is the Fermi distribution function, $\varepsilon_{\alpha}\left(\varepsilon_{\beta}\right)$ and $|\alpha\rangle(|\beta\rangle)$ represent the eigenenergy and the eigenstate of the system, $v_{x}=-(i / \hbar)[x, H]$ is the velocity operator, and $\eta$ is the phenomenological broadening which is set to $3 \mathrm{meV}$ in the following calculations. The optical absorption intensity at photon energies $\hbar \omega$ is related to the real part of $\sigma(\omega)$. The transmission of light incident perpendicular to two-dimensional system is given by $\underline{58}$

$$
T=\left|1+\frac{2 \pi}{c} \sigma_{x x}(\omega)\right|^{-2} \approx 1-\frac{4 \pi}{c} \operatorname{Re} \sigma_{x x}(\omega) .
$$

\section{RESULTS AND DISCUSSIONS}

\section{A. Band structure}

The band dispersion of TBG can be intuitively understood in terms of coupled four Dirac cones centered at the valleys $K^{(l)}$ and $K^{(l)}(l=1,2)$. Figure 3 shows the schematic band structures of TBG in the extended Brillouin zone along the line $K^{\prime(2)}-K^{\prime(1)}-K^{(2)}-K^{(1)}$, where Dashed and solid dispersion represent the energy bands of the layer 1 and 2, respectively. The interlayer coupling gives rise to band anticrossing at the intersection, and the resultant energy band is characterized by saddle points accompanied by the van Hove singularity in the density of states. The saddle points are classified into (i), (ii) and (iii) as shown in Fig. 3 where (i) and (ii) result from the band intersection of different layers, while (iii) originates from the original monolayer's band structure. In the limit of $\theta \rightarrow 0, K^{(1)}$ and $K^{(2)}$ (equivalently, $K^{\prime(1)}$ and $\left.K^{\prime(2)}\right)$ get closer, so that (i) approaches the Dirac points, while (ii) goes to the midpoint between $K$ and $K^{\prime}$ in the high energy region. (iii) remains at the constant energy.

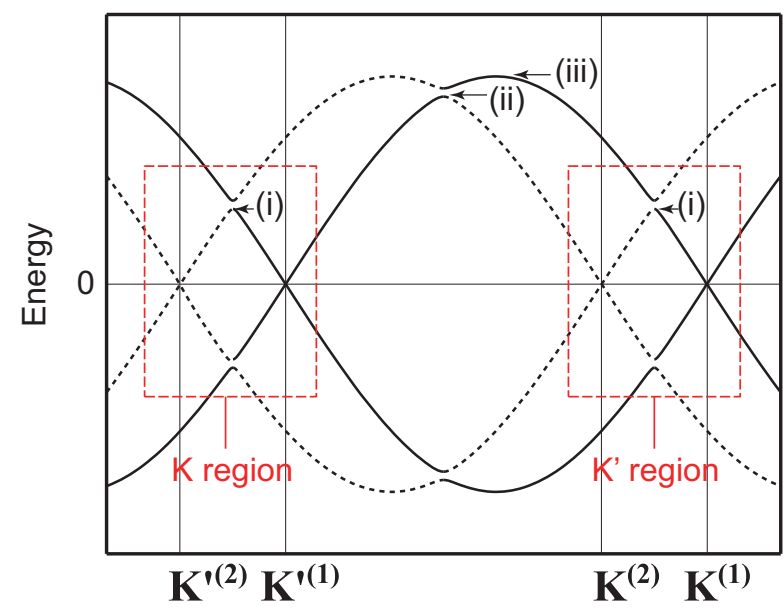

FIG. 3: Schematic band structures of TBG in the extended zone scheme along the line $K^{\prime(2)}-K^{\prime(1)}-K^{(2)}-K^{(1)}$. Symbols (i), (ii) and (iii) indicate the saddle points.

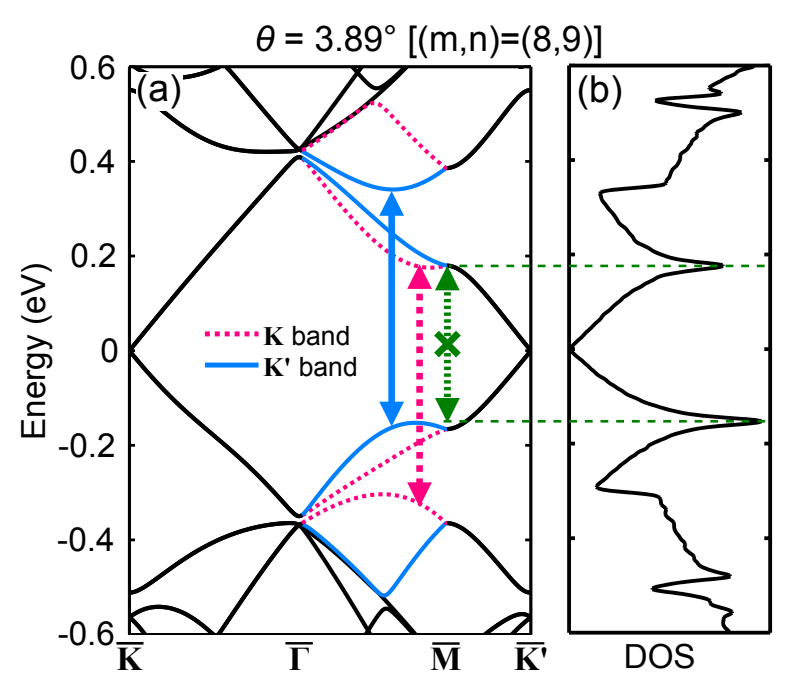

FIG. 4: (a) Band structure and (b) density of states of TBG with $\theta=3.89^{\circ}$. Solid (blue) and dashed (pink) arrows represent the excitation corresponding to the major peaks in the optical absorption, and green dotted arrow is a process optically forbidden (see text).

Figures 4(a) and 4(b) show the band structure and the density of state (DOS), respectively, of TBG with $\theta=3.89^{\circ}[(m, n)=(8,9)]$ actually calculated by the tight-binding model Eq. (7). The band structure can be viewed as monolayer's Dirac cone folded into the superlattice Brillouin zone with some band anticrossing at the zone corner $\frac{7,11,12,59-62}{6}$ The lowest band exhibits a linear dispersion near $\bar{K}$ and $\bar{K}^{\prime}$, while we see a large splitting near the $\bar{M}$ point in energy from $\pm 0.2 \mathrm{eV}$ to $\pm 0.4 \mathrm{eV}$, respectively, corresponding to the band anticrossing (i) in 


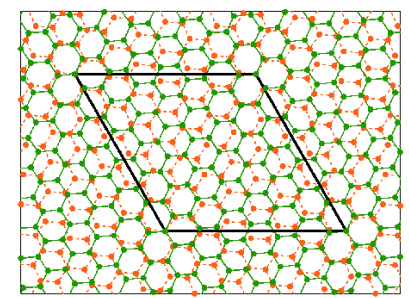

(a) $\theta=9.43^{\circ}[(m, n)=(3,4)]$

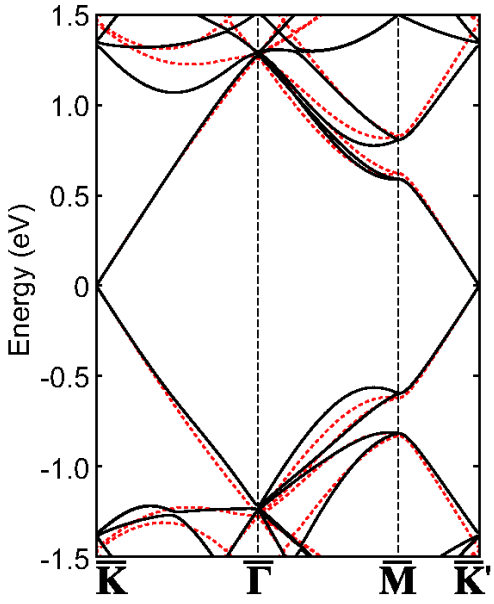

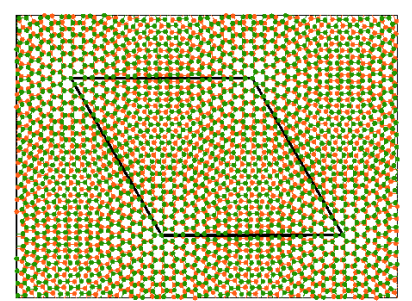
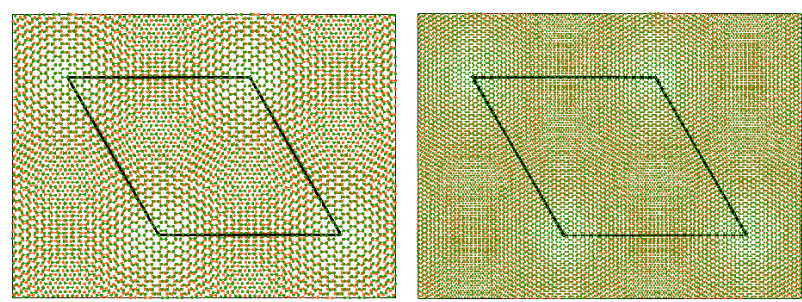

(b) $\theta=3.89^{\circ}[(m, n)=(8,9)]$

(c) $\theta=2.65^{\circ}[(m, n)=(12,13)]$

(d) $\theta=1.47^{\circ}[(m, n)=(22,23)]$
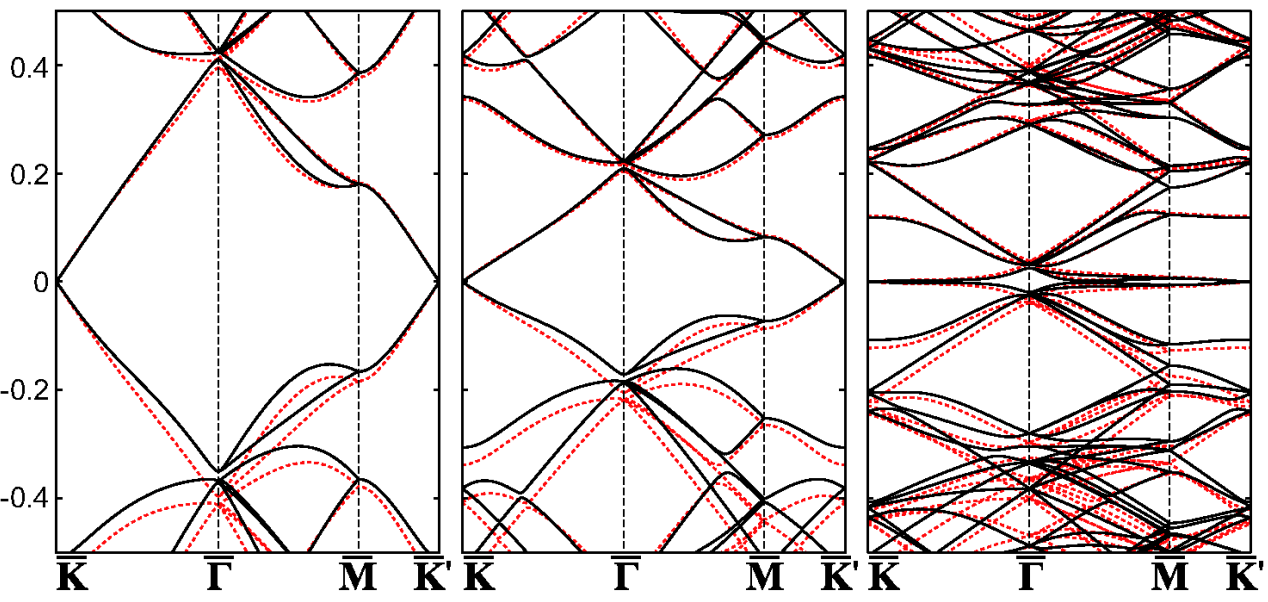

FIG. 5: Atomic structures (top) and band structures (bottom) of TBGs with (a) $\theta=9.43^{\circ}$, (b) $3.89^{\circ}$, (c) $2.65^{\circ}$, and (d) $1.47^{\circ}$, calculated by the tight-binding model (solid black lines) and the effective continuum model (dashed red lines). Dirac point energy is set to zero.

Fig. 3 $3,62,63$ The DOS has a sharp peak around $\pm 0.2 \mathrm{eV}$, which is associated with the saddle point near the $\bar{M}$ point.

Each energy band can be classified into either of those originating from monolayer's $K$ region (i.e., $K^{(1)}$ and $K^{(2)}$ ) or those from $K^{\prime}$ region (i.e., $K^{\prime(1)}$ and $K^{\prime(2)}$ ), because two valleys are hardly mixed by the interlayer interaction in this small rotation angle. Here $K$ and $K^{\prime}$ should not be confused with $\bar{K}$ and $\bar{K}^{\prime}$ for the folded Brillouin zone. The monolayer's band near $K$ and that near $K^{\prime}$ are independently folded into the same Brillouin zone without mixing with each other. Indeed, the lowest band in Fig. 4(a) is composed of nearly degenerate branches, where dashed (pink) and solid (blue) lines are the bands from $K$ and $K^{\prime}$, respectively. These two bands are degenerate along $\bar{K}-\bar{\Gamma}$ and $\bar{M}-\bar{K}^{\prime}$, reflecting the $C_{2}$ symmetry in the real-space lattice structure ${ }^{62}$.

Figure 5 shows the energy bands of TBGs with different rotation angles from $\theta=9.43^{\circ}$ down to $1.47^{\circ}$. The structures are similar to each other while the overall energy scale shrinks as the rotation angle decreases, roughly in proportion to the size of the folded Brillouin zone. The width of splitting at the $\bar{M}$ point is about $0.2 \mathrm{eV}$ in every case, which is of the order of the interlayer coupling $V_{p p \sigma}^{0}$. In small rotation angles less than $2^{\circ}$, the energy scale of the folded Dirac cone becomes comparable to the band splitting so that the band velocity near the Dirac cone is significantly reduced from the monolayer's, $2,11-16$ In Fig. 5, we also plot the band energies calculated by the effective continuum model Eq. (17) as dashed (red) lines, to be compared with solid (black) curves obtained by the original tight-binding model. We see the low-energy band structure agrees quite well, except that the effective model fails to reproduce a small electron hole asymmetry in the original model, since it assumes the symmetric Dirac cone for the intralayer Hamiltonian.

Figure [6]shows the DOS of TBGs with various rotation angles $0^{\circ}<\theta<30^{\circ}$ with (a) wide and (b) narrow energy ranges. To each curve, we append the DOS of uncoupled bilayer graphene (i.e., twice of monolayer's) as a light gray line. We observe a number of characteristic peaks associated with van Hove singularities (i), (ii) and (iii) argued in Fig. 3. As the rotation angle increases, the peaks (i) move away from the Dirac points, and the peaks (ii) move towards the Dirac points, while the peaks (iii) stay at almost constant energy. In small rotation angles, we see a number of additional peaks since the interlayer coupling of the higher order in the Moiré wave number becomes significant. 
(a)

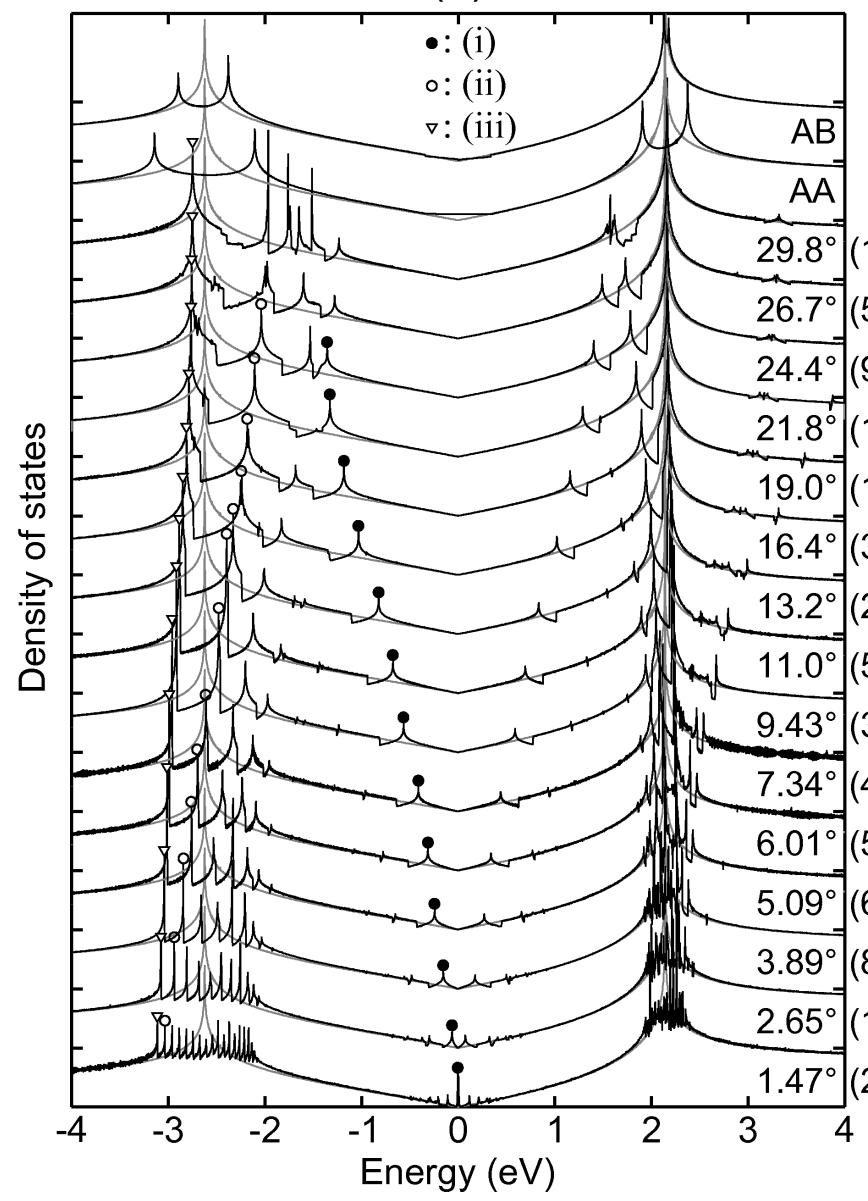

(b)

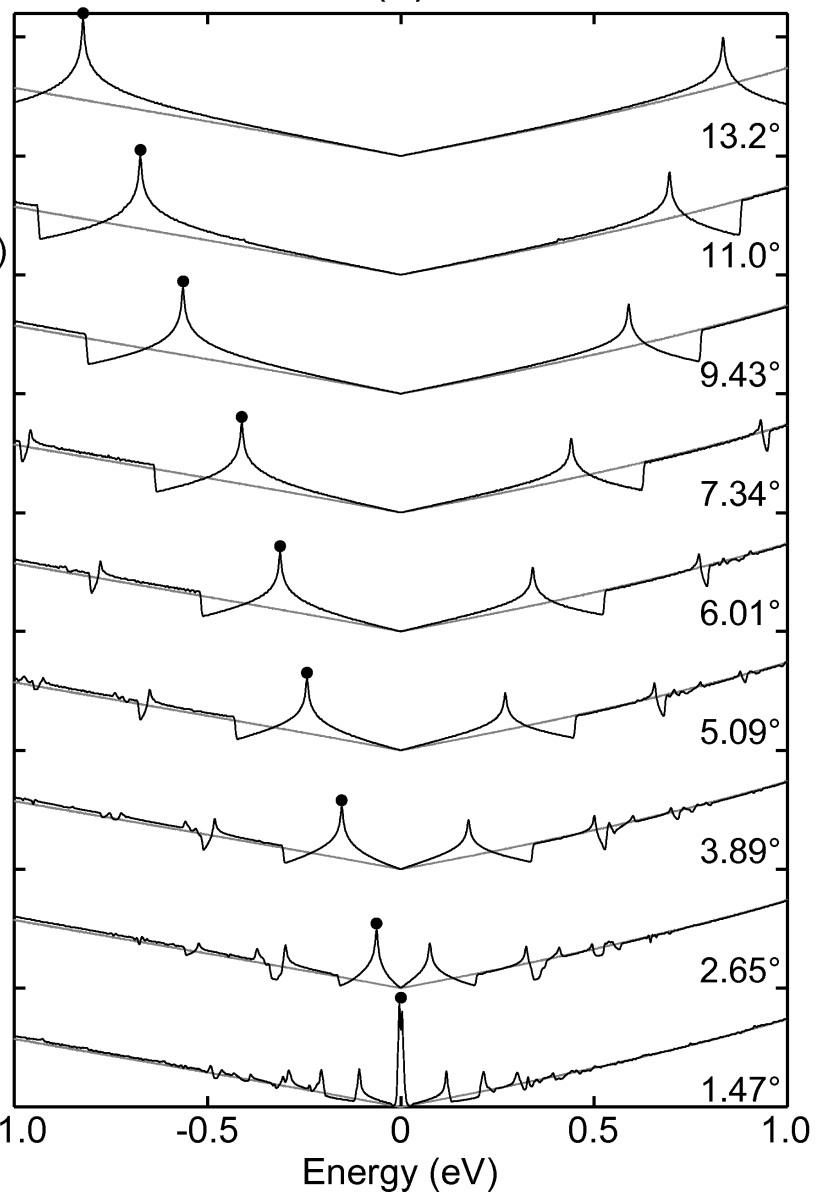

FIG. 6: DOS of TBGs with various rotation angles $0^{\circ}<\theta<30^{\circ}$ in (a) wide and (b) narrow ranges of the energy. The DOS of uncoupled bilayer graphene (i.e., twice of the monolayer's DOS) is shown as light gray lines. Peaks marked with symbols correspond to three different types of van Hove singularity (see text).

\section{B. Optical absorption}

In Fig. 7 we plot the dynamical conductivity of TBG with $\theta=3.89^{\circ}$ calculated by the tight-binding model. The conductivity is plotted in units of

$$
\sigma_{\text {mono }}=\frac{g_{v} g_{s}}{16} \frac{e^{2}}{\hbar}
$$

which is the universal dynamical conductivity of monolayer graphene at linear band regime, where $g_{s}=2$ and $g_{v}=2$ are the spin and valley $\left(K, K^{\prime}\right)$ degeneracy, respectively $23.35-38$ The spectrum is characterized by a peak around $0.5 \mathrm{eV}$, and considerable reduction right below the peak energy. Otherwise the conductivity is close to $2 \sigma_{\text {mono. }}$. The top and bottom panels numbered from (1) to (4) show spectral weight maps at specific photon energies, which highlight the wave vectors that contribute to the optical transition. A sudden rise in the conductivity between (3) and (4) is due to bright spots near $\bar{M}$ point in the weight map. This actually corresponds to the transition from the saddle point in the lowest valence band to the second conduction band, which is marked with a solid (blue) arrow in the band diagram in Fig. 4(a). The similar transition indicated by dashed (pink) arrow occurs at slightly higher energy due to the electron-hole asymmetry. Below the peak energy, the dynamical conductivity is significantly reduced and becomes lower than $2 \sigma_{\text {mono }}$, because the number of available states largely decreases due to the band anticrossing.

It should be noted that the transition does not occur between the saddle points of the lowest conduction and valence bands [dotted (green) arrow in Fig. 世(a)], because it is optically forbidden in this particular system. This can be clearly explained by the effective continuum model as following. The lowest electron and hole states at $\bar{M}$ point, which are connected by dotted arrow in Fig. 4, are an electron-hole pair related by the symmetry of Eq. (20), and thus these wavefunctions are written as $\psi$ and $\hat{\Sigma} \psi^{*}$. The matrix element of $v_{x}$ between the two states 

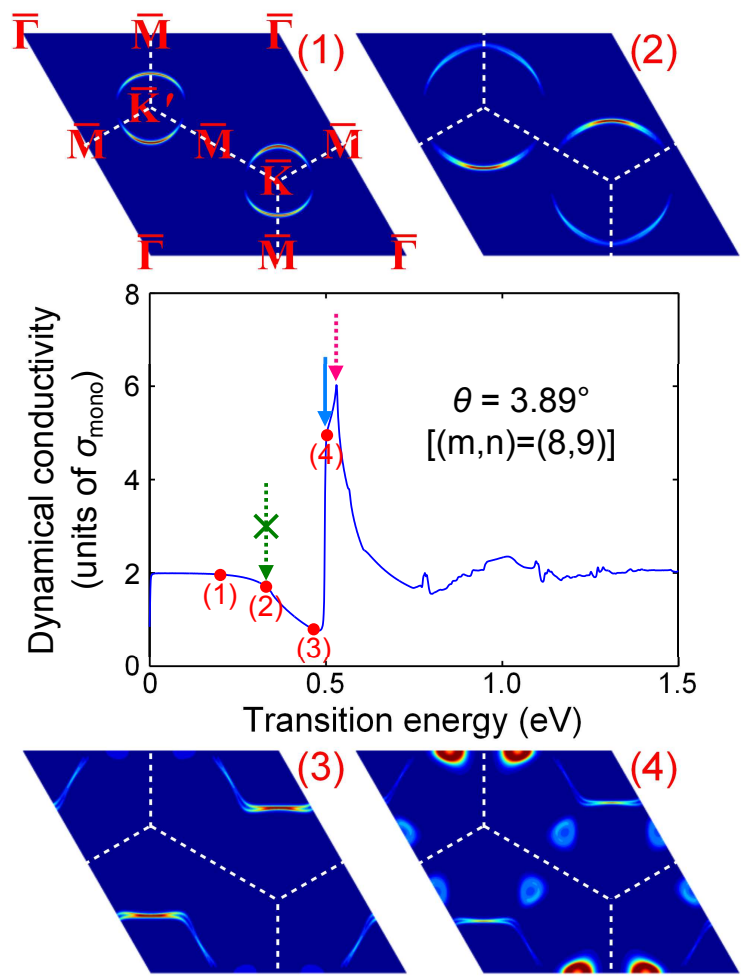

FIG. 7: (Middle panel) Dynamical conductivity of TBG with $\theta=3.89^{\circ}$ as a function of the transition energy. Arrows indicate the excitation energies of the transitions shown in Fig. 4(a). (Top and bottom panels) Spectral weight maps in the superlattice Brillouin zone at several transition energies.

is obviously zero, because

$$
\begin{aligned}
& \left\langle\hat{\Sigma} \psi^{*}\left|v_{x}\right| \psi\right\rangle=\left(\begin{array}{c}
\psi_{4}^{*} \\
\psi_{3}^{*} \\
-\psi_{2}^{*} \\
-\psi_{1}^{*}
\end{array}\right)^{\dagger}\left(\begin{array}{cc}
v \sigma_{x} & 0 \\
0 & v \sigma_{x}
\end{array}\right)\left(\begin{array}{l}
\psi_{1} \\
\psi_{2} \\
\psi_{3} \\
\psi_{4}
\end{array}\right) \\
& =v\left(\psi_{4} \psi_{2}+\psi_{3} \psi_{1}-\psi_{2} \psi_{4}-\psi_{1} \psi_{3}\right)=0,
\end{aligned}
$$

and thus the transition is optically inactive. This symmetry does not limit the optical selection rule at other points than $\bar{M}$ [the origin of the wave number in the effective Hamiltonian Eq. [17]), since $\psi$ and $\hat{\Sigma} \psi^{*}$ generally reside at different Bloch wave vectors and are not connected by the optical transition.

In Fig. 8(a), we plot the optical absorption spectra at various rotation angles in a wide frequency range. The spectrum exhibits characteristic conductivity peaks ranging from terahertz to ultraviolet frequencies. The peaks are again classified into three groups similarly to the density of states. When the rotation angle increases from $0^{\circ}$ to $30^{\circ}$, the peak (i) [(ii)] moves to higher (lower) energies, while the peak (iii) remains unchanged. Figure 8(b) shows magnified plots of the low-frequency range for several small angles. There the spectrum is charac- terized by a single peak belonging to group (i) similarly to Fig. (7) and its transition energy monotonically shifts with the rotation angle. In $\theta=1.47^{\circ}$, the spectrum exhibits a complicated structure in accordance with the strong band deformation observed in Fig. 5. In Fig. 8(b), we also present the spectrum of the effective continuum model as dashed (red) curves, to be compared with the original tight-binding calculation. The results agree quite well except for the peak splitting due to the electron-hole asymmetry, which is pronounced in $\theta \gtrsim 10^{\circ}$.

As argued in Sec. IIA the rigorous superlattice period $L$ in Eq. (3) discontinuously changes depending on the commensurability of the lattice periods. Nevertheless, the DOS in Fig. 6 and the optical absorption spectrum in Fig. 8 show that the peak structure almost continuously evolves with the rotation angle, suggesting that the exact lattice commensurability is not quite important for the physical property. Although we cannot rigorously handle incommensurate TBGs due to infinite unit cell size, its optical spectrum should be approximated by interpolating those of commensurate TBGs with similar rotation angles. The only property in which the actual lattice period $L$ matters is found as tiny peaks in the conductivity indicated as dashed (blue) circles in Fig. 8, which exist only when $|m-n|>1$. They are related to the transition at the corner of the exact superlattice Brillouin zone.

\section{Effect of lattice displacement}

The electronic structure depends on the lattice displacement $\boldsymbol{\delta}$, particularly when the superlattice period $L$ is comparable to the atomic scale. This effect is maximum at $\theta=0$, where the lattice constant $L$ coincides with $a$, and the AA stacking is transformed to the AB stacking by a translation $\delta$. The TBG with $(m, n)=(1,2)\left(\theta=21.8^{\circ}\right)$ has the next smallest primitive unit cell of $L=\sqrt{7} a$. Here we focus on two distinct TBGs of $(m, n)=(1,2)$ with $\boldsymbol{\delta}=0$ and $\left(\mathbf{a}_{1}^{(2)}+\mathbf{a}_{2}^{(2)}\right) / 3$, shown in Figs. 9(a) and (b), respectively, to investigate the effect of the relative translation on the optical absorption. These two structures can be also described by two different rotation angles $\theta$ and $60^{\circ}-\theta$. Figures 9(c) and (d) show the electronic structure and the dynamical conductivity, respectively, of the two different TBGs. We observe that the energy bands are almost equivalent, while there is a tiny difference in the structure near $\bar{M}$ point of which the energy scale is a few tens of meV. In the dynamical conductivity, this is reflected in a small difference in the peak structure near the energy of $3 \mathrm{eV}$. The inset in Fig. 9(c) magnifies the band structure in the vicinity the Dirac points. We observe a difference of the energy scale about a few $\mathrm{meV}$, where one takes the form like AA-bilayer and the other like AB-bilayer, while it gives no noticeable difference in the dynamical conductivity in Fig. 9(d). The dependence on the lattice displacement becomes even smaller as the superlattice period $L$ becomes larger, and is completely absent in 

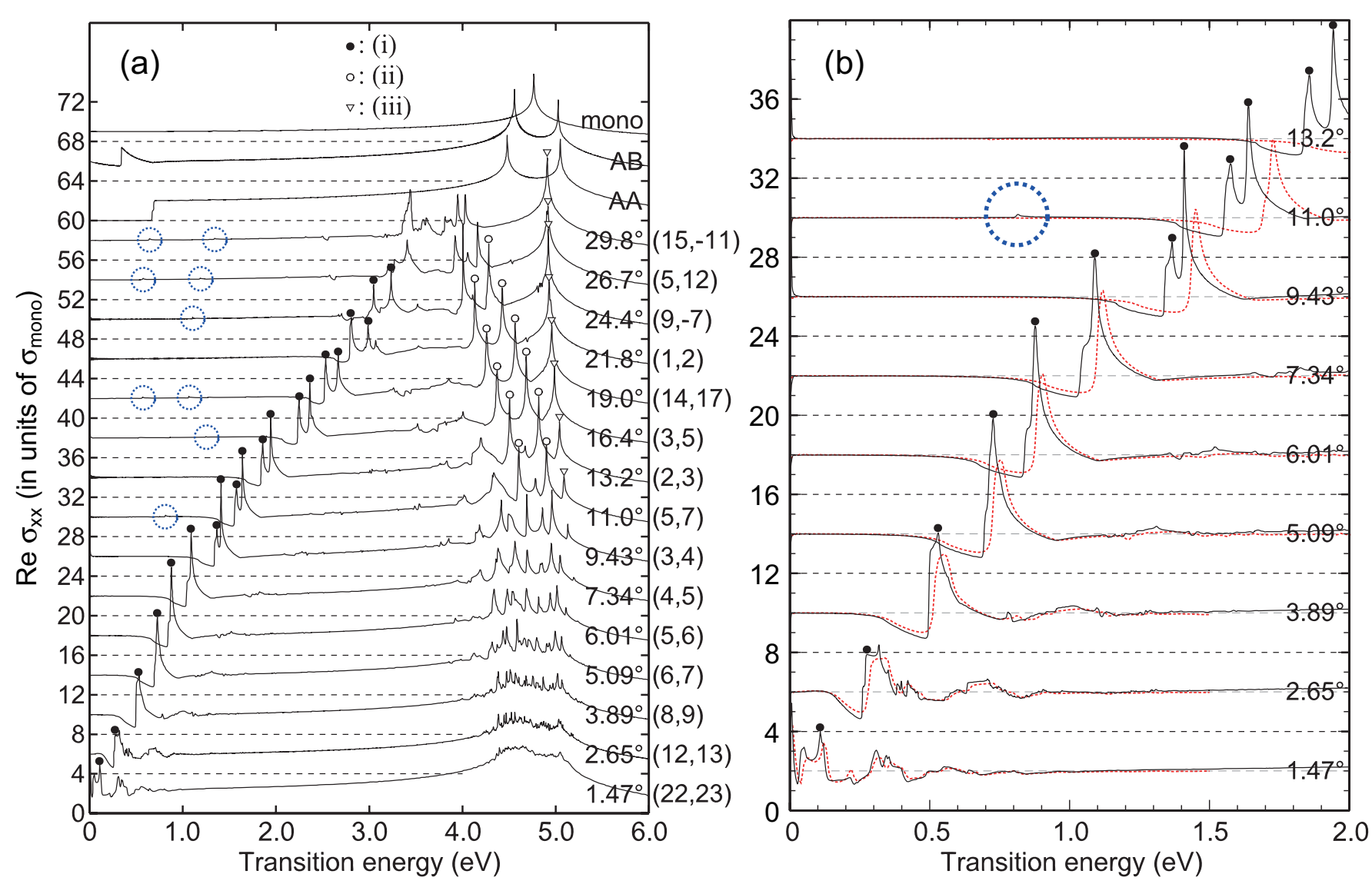

FIG. 8: Dynamical conductivities of TBGs with various rotation angles in (a) wide and (b) narrow frequency ranges, calculated by the tight-binding model (solid black lines) and the effective continuum model (dashed red lines, only for (b)). Peaks marked with symbols represent the excitations associated with the van Hove singularity in Fig. 6 Dashed (blue) circles indicate the tiny peaks which appears only when the actual lattice period $L$ is larger than the Moiré period $L_{\mathrm{M}}$ (see text).

any incommensurate angles. Thus, concerning the energy range of interest, we conclude that the optical spectrum of TBG does not depends much on the lattice displacement, except for $\theta=0$.

\section{CONCLUSION}

We theoretically investigated the optical absorption properties of TBGs with various stacking geometries using the tight-binding model and the effective continuum model. We showed that the spectrum is characterized by series of absorption peaks associated with the van Hove singularities in the band structure, and the peak energies systematically shift in changing the rotation angle. The optical spectrum almost continuously evolves in changing the rotation angle regardless of the rigorous commensurability between two layers, suggesting that the optical absorption measurement provides a convenient way to identify the rotation angle of TBG. We developed the effective continuum model based on the tight-binding model used here, and demonstrated that it well reproduces the low-energy band structure and the dynamical conductivity of the tight-binding model for $\theta<10^{\circ}$, and it also explains the optical selection rule analytically in terms of the symmetry of the effective Hamiltonian.

\section{ACKNOWLEDGMENTS}

This project has been funded by Grant-in-Aid for Research Activity Start-up No. 23840004 (PM), Grant-inAid for Scientific Research No. 24740193 (MK) from Japan Society for the Promotion of Science (JSPS), and by JST-EPSRC Japan-UK Cooperative Programme Grant No. EP/H025804/1. P. M. acknowledges the Supercomputer Center, Institute for Solid State Physics, University of Tokyo for the use of the facilities (Project No. ID: H23-D-0009). 


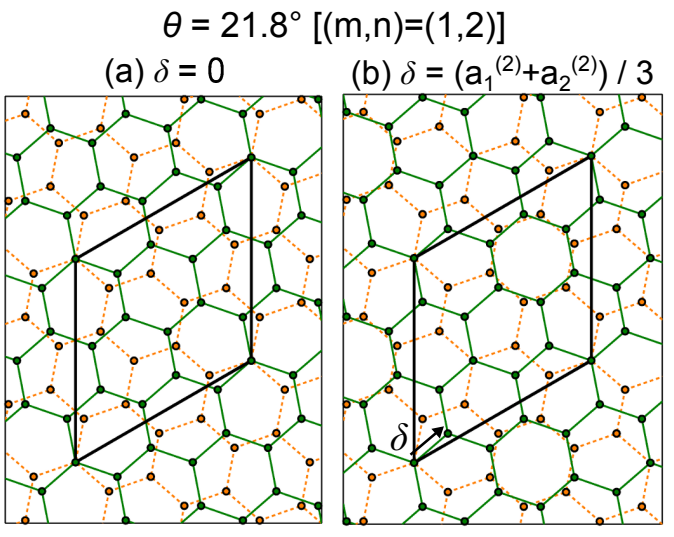

(c) Band structure

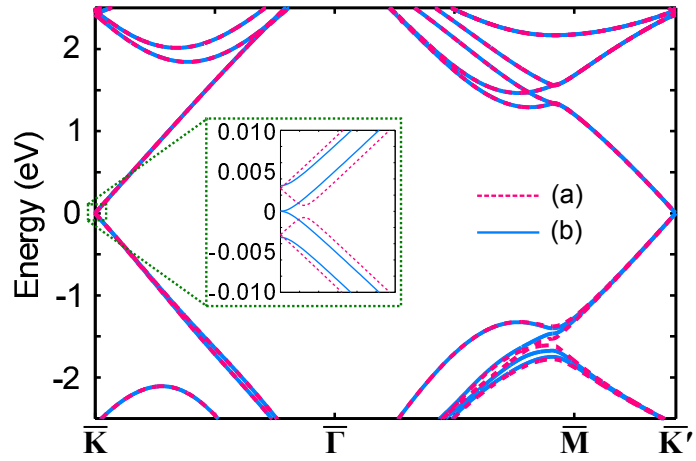

(d) Dynamical conductivity

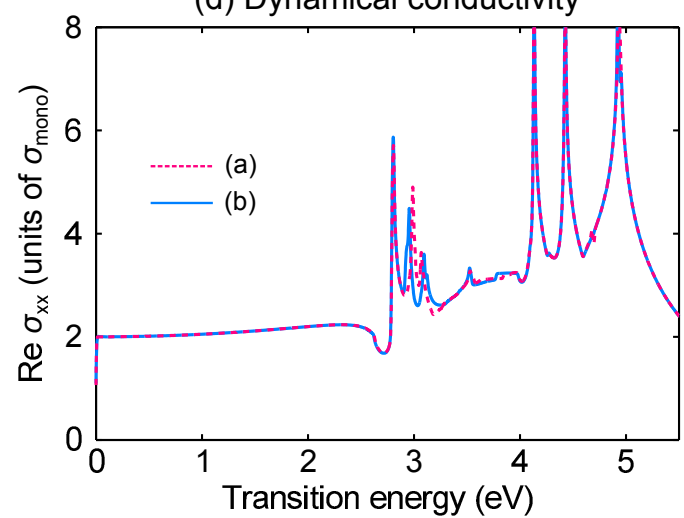

FIG. 9: Atomic structures of TBGs of $\theta=21.8^{\circ}$ with different translation vectors, (a) $\boldsymbol{\delta}=0$ and (b) $\left(\mathbf{a}_{1}^{(2)}+\mathbf{a}_{2}^{(2)}\right) / 3$. (c) Band structures and (d) dynamical conductivities of the two distinct TBGs.

\section{Appendix A: Derivation of effective interlayer coupling}

Here we derive the effective interlayer matrix element Eq. (16) for TBG with small rotation angles. The matrix element of the Hamiltonian Eq. (17) between the single layer bases on the different layers is explicitly written as

$$
\begin{aligned}
& \left\langle\mathbf{k}^{\prime}, X_{2}^{\prime}|H| \mathbf{k}, X_{1}\right\rangle \\
& =\frac{1}{N} \sum_{\mathbf{R}_{X_{2}^{\prime}}, \mathbf{R}_{X_{1}}}-t\left(\mathbf{R}_{X_{2}^{\prime}}-\mathbf{R}_{X_{1}}\right) \exp \left[-i \mathbf{k}^{\prime} \cdot \mathbf{R}_{X_{2}^{\prime}}+i \mathbf{k} \cdot \mathbf{R}_{X_{1}}\right] \\
& =\frac{1}{N} \sum_{\mathbf{R}_{X_{2}^{\prime}}, \mathbf{R}_{X_{1}}}-t\left(\mathbf{R}_{X_{2}^{\prime}}-\mathbf{R}_{X_{1}}\right) \\
& \quad \times \exp \left[-i \overline{\mathbf{k}} \cdot\left(\mathbf{R}_{X_{2}^{\prime}}-\mathbf{R}_{X_{1}}\right)\right] \exp \left[-i \Delta \mathbf{k} \cdot \frac{\mathbf{R}_{X_{2}^{\prime}}+\mathbf{R}_{X_{1}}}{2}\right] .
\end{aligned}
$$

where

$$
\overline{\mathbf{k}}=\frac{\mathbf{k}+\mathbf{k}^{\prime}}{2}, \quad \Delta \mathbf{k}=\mathbf{k}^{\prime}-\mathbf{k} .
$$

Since the Moire lattice constant $L_{\mathrm{M}}$ is much larger than $a$ in small rotation angles, we only need to consider $\mathbf{k}$ and $\mathbf{k}^{\prime}$ which are close to each other, or $|\Delta \mathbf{k}| \ll 2 \pi / a$. Then the last exponential term in Eq. (A1) slowly varies in the lattice position, while other terms change in the atomic length scale. To separate out the long wave component, we introduce a smoothing function $g(\mathbf{r})$ which satisfies the following conditions: ${ }^{57} g(\mathbf{r})$ varies in $\mathbf{r}$ in an intermediate length scale $l_{g}$, which is much larger than the lattice constant $a$, but much smaller than the Moiré superlattice period $L_{\mathrm{M}} . g(\mathbf{r})$ is a peak centered at $\mathbf{r}=0$, and rapidly decays in $\mathbf{r} \gtrsim l_{g}$. The area is normalized as

$$
\int g(\mathbf{r}) d \mathbf{r}=\Omega_{\mathrm{M}}
$$

where $\Omega_{\mathrm{M}}$ is the Moiré superlattice unit cell, and the integral is taken over the whole system area unless otherwise stated. Almost equivalently, we have

$$
\sum_{\mathbf{R}_{X}} g\left(\mathbf{r}-\mathbf{R}_{X}\right)=\frac{\Omega_{\mathrm{M}}}{\Omega_{0}}
$$

where $X$ is either of $A_{1}, B_{1}, A_{2}$ or $B_{2}$, and $\Omega_{0}=\left|\mathbf{a}_{1} \times \mathbf{a}_{2}\right|$ is the area of monolayer's unit cell.

Using Eq. (A4), the matrix element Eq. (A1) is written as

$$
\begin{aligned}
& \left\langle\mathbf{k}^{\prime}, X_{2}^{\prime}|H| \mathbf{k}, X_{1}\right\rangle \\
& =\frac{1}{N} \sum_{\mathbf{R}_{X_{2}^{\prime}}, \mathbf{R}_{X_{1}}}\left[\frac{1}{\Omega_{\mathrm{M}}} \int g\left(\mathbf{r}-\mathbf{R}_{X_{1}}\right) d \mathbf{r}\right]\left[-t\left(\mathbf{R}_{X_{2}^{\prime}}-\mathbf{R}_{X_{1}}\right)\right] \\
& \quad \times \exp \left[-i \overline{\mathbf{k}} \cdot\left(\mathbf{R}_{X_{2}^{\prime}}-\mathbf{R}_{X_{1}}\right)\right] \exp \left[-i \Delta \mathbf{k} \cdot \frac{\mathbf{R}_{X_{2}^{\prime}}+\mathbf{R}_{X_{1}}}{2}\right] .
\end{aligned}
$$

Now the argument $\left(\mathbf{R}_{X_{2}^{\prime}}+\mathbf{R}_{X_{1}}\right) / 2$ in the last can be replaced with $\mathbf{R}_{X_{1}}$, because the last exponential term varies slowly with the length scale of $L_{\mathrm{M}}$, and also the hopping integral $t\left(\mathbf{R}_{X_{2}^{\prime}}-\mathbf{R}_{X_{1}}\right)$ occurs only in the atomic scale distance. This is further replaced with $\mathbf{r}$, since the 
smoothing factor $g\left(\mathbf{r}-\mathbf{R}_{X_{1}}\right)$ works as the delta function for the slowly varying function with the scale $L_{\mathrm{M}}$. By including this, we obtain

$$
\begin{aligned}
& \left\langle\mathbf{k}^{\prime}, X_{2}^{\prime}|H| \mathbf{k}, X_{1}\right\rangle \\
& \approx \frac{1}{N \Omega_{\mathrm{M}}} \int d \mathbf{r} e^{-i \Delta \mathbf{k} \cdot \mathbf{r}} \sum_{\mathbf{R}_{X_{1}}} g\left(\mathbf{r}-\mathbf{R}_{X_{1}}\right) \\
& \quad \times \sum_{\mathbf{R}_{X_{2}^{\prime}}}\left[-t\left(\mathbf{R}_{X_{2}^{\prime}}-\mathbf{R}_{X_{1}}\right)\right] \exp \left[-i \overline{\mathbf{k}} \cdot\left(\mathbf{R}_{X_{2}^{\prime}}-\mathbf{R}_{X_{1}}\right)\right] \\
& =\frac{1}{\Omega_{\mathrm{M}}} \int_{\Omega_{\mathrm{M}}} d \mathbf{r} e^{-i \Delta \mathbf{k} \cdot \mathbf{r}} U_{X_{2}^{\prime} X_{1}}[\overline{\mathbf{k}}, \boldsymbol{\delta}(\mathbf{r})]
\end{aligned}
$$

where we used Eq. (A44) and $\int d \mathbf{r} /\left(N \Omega_{0}\right)=\int_{\Omega_{\mathrm{M}}} d \mathbf{r} / \Omega_{\mathrm{M}}$. The last equation is the final result of Eq. (16).
1 C. Berger, Z. Song, X. Li, X. Wu, N. Brown, C. Naud, D. Mayou, T. Li, J. Hass, A. N. Marchenkov, et al., Science 312, 1191 (2006).

2 Z. Ni, Y. Wang, T. Yu, Y. You, and Z. Shen, Phys. Rev. B 77, 235403 (2008).

3 Z. Yan, Z. Peng, Z. Sun, J. Yao, Y. Zhu, Z. Liu, P. M. Ajayan, and J. M. Tour, ACS Nano 5, 8187 (2011).

4 L. Xie, H. Wang, C. Jin, X. Wang, L. Jiao, K. Suenaga, and H. Dai, J. Am. Chem. Soc. 133, 10394 (2011).

5 R. Zhao, Y. Zhang, T. Gao, Y. Gao, N. Liu, L. Fu, and Z. Liu, Nano Res. 4, 712 (2011).

6 J. Hass, R. Feng, J. Millan-Otoya, X. Li, M. Sprinkle, P. First, W. De Heer, E. Conrad, and C. Berger, Phys. Rev. B 75, 214109 (2007).

7 J. Hass, F. Varchon, J. Millan-Otoya, M. Sprinkle, N. Sharma, W. de Heer, C. Berger, P. First, L. Magaud, and E. Conrad, Phys. Rev. Lett. 100, 125504 (2008).

8 G. Li, A. Luican, J. Dos Santos, A. Neto, A. Reina, J. Kong, and E. Andrei, Nat. Phys. 6, 109 (2009).

9 D. Miller, K. Kubista, G. Rutter, M. Ruan, W. de Heer, P. First, and J. Stroscio, Phys. Rev. B 81, 125427 (2010).

10 A. Luican, G. Li, A. Reina, J. Kong, R. Nair, K. Novoselov, A. Geim, and E. Andrei, Phys. Rev. Lett. 106, 126802 (2011).

11 J. Lopes dos Santos, N. Peres, and A. Castro Neto, Phys. Rev. Lett. 99, 256802 (2007).

12 E. Morell, J. Correa, P. Vargas, M. Pacheco, and Z. Barticevic, Phys. Rev. B 82, 121407 (2010).

13 S. Shallcross, S. Sharma, E. Kandelaki, and O. Pankratov, Phys. Rev. B 81, 165105 (2010).

14 G. Trambly De Laissardière, D. Mayou, and L. Magaud, Nano Lett. 10, 804 (2010).

15 R. Bistritzer and A. MacDonald, PNAS 108, 12233 (2011).

16 J. M. B. Lopes dos Santos, N. M. R. Peres, and A. H. Castro Neto, Phys. Rev. B 86, 155449 (2012).

17 Z. Ni, L. Liu, Y. Wang, Z. Zheng, L. Li, T. Yu, and Z. Shen, Phys. Rev. B 80, 125404 (2009).

18 A. Righi, S. Costa, H. Chacham, C. Fantini, P. Venezuela, C. Magnuson, L. Colombo, W. Bacsa, R. Ruoff, and M. Pimenta, Phys. Rev. B 84, 241409 (2011).

19 K. Sato, R. Saito, C. Cong, T. Yu, and M. S. Dresselhaus, Phys. Rev. B 86, 125414 (2012).

20 Y. Wang, Z. Ni, L. Liu, Y. Liu, C. Cong, T. Yu, X. Wang, D. Shen, and Z. Shen, ACS Nano 4, 4074 (2010).

21 T. Ohta, T. E. Beechem, J. T. Robinson, and G. L. Kellogg, Phys. Rev. B 85, 075415 (2012).
${ }^{22}$ X. Zou, J. Shang, J. Leaw, Z. Luo, L. Luo, C. La-o vorakiat, L. Cheng, S. A. Cheong, H. Su, J.-X. Zhu, et al., Phys. Rev. Lett. 110, 067401 (2013).

${ }^{23}$ R. Nair, P. Blake, A. Grigorenko, K. Novoselov, T. Booth, T. Stauber, N. Peres, and A. Geim, Science 320, 1308 (2008).

24 Z. Li, E. Henriksen, Z. Jiang, Z. Hao, M. Martin, P. Kim, H. Stormer, and D. N. Basov, Nat. Phys. 4, 532 (2008).

${ }^{25}$ K. F. Mak, M. Y. Sfeir, Y. Wu, C. H. Lui, J. A. Misewich, and T. F. Heinz, Phys. Rev. Lett. 101, 196405 (2008).

${ }^{26}$ L. Zhang, Z. Li, D. Basov, M. Fogler, Z. Hao, and M. Martin, Phys. Rev. B 78, 235408 (2008).

27 E. Henriksen, Z. Jiang, L.-C. Tung, M. Schwartz, M. Takita, Y.-J. Wang, P. Kim, and H. Stormer, Phys. Rev. Lett. 100, 87403 (2008).

28 Z. Li, E. Henriksen, Z. Jiang, Z. Hao, M. C. Martin, P. Kim, H. Stormer, and D. N. Basov, Phys. Rev. Lett. 102, 37403 (2009).

29 Y. Zhang, T.-T. Tang, C. Girit, Z. Hao, M. C. Martin, A. Zettl, M. F. Crommie, Y. R. Shen, and F. Wang, Nature 459, 820 (2009).

30 M. Orlita, C. Faugeras, J. M. Schneider, G. Martinez, D. K. Maude, and M. Potemski, Phys. Rev. Lett. 102, 166401 (2009).

31 A. Kuzmenko, E. van Heumen, D. van der Marel, P. Lerch, P. Blake, K. Novoselov, and A. Geim, Phys. Rev. B 79, 115441 (2009).

32 A. Kuzmenko, I. Crassee, D. Van der Marel, P. Blake, and K. Novoselov, Phys. Rev. B 80, 165406 (2009).

33 K. F. Mak, M. Y. Sfeir, J. A. Misewich, and T. F. Heinz, PNAS 107, 14999 (2010).

${ }^{34}$ K. F. Mak, J. Shan, and T. F. Heinz, Phys. Rev. Lett. 104, 176404 (2010).

35 T. Ando, Y. Zheng, and H. Suzuura, J. Phys. Soc. Jpn. 71, 1318 (2002).

36 V. Gusynin and S. Sharapov, Phys. Rev. B 73, 245411 (2006).

37 V. Gusynin, S. Sharapov, and J. Carbotte, Phys. Rev. Lett. 96, 256802 (2006).

38 V. Gusynin, S. Sharapov, and J. Carbotte, Phys. Rev. Lett. 98, 157402 (2007).

39 D. Abergel and V. Fal'ko, Phys. Rev. B 75, 155430 (2007).

40 L. Falkovsky and S. Pershoguba, Phys. Rev. B 76, 153410 (2007).

41 M. Koshino and T. Ando, Phys. Rev. B 77, 115313 (2008).

42 M. Koshino and T. Ando, Solid State Commun. 149, 1123 
(2009).

43 N. Peres, Rev. Mod. Phys. 82, 2673 (2010).

44 M. Koshino, New J. Phys. 15, 015010 (2013).

45 Z. Chen and X. Wang, Phys. Rev. B 83, 081405 (2011).

46 E. Mele, Phys. Rev. B 81, 161405 (2010).

47 E. Mele, J. Phys. D: Appl. Phys. 45, 154004 (2012).

48 T. A. Green and J. Weigle, Helv. Phys. Acta 21, 217 (1948).

49 T. Nakanishi and T. Ando, J. Phys. Soc. Jpn. 70, 1647 (2001).

50 S. Uryu, Phys. Rev. B 69, 075402 (2004).

51 J. Slater and G. Koster, Phys. Rev. 94, 1498 (1954).

${ }^{52}$ M. Kindermann and P. First, Phys. Rev. B 83, 045425 (2011).

53 J. McClure, Phys. Rev. 104, 666 (1956).

54 D. DiVincenzo and E. Mele, Phys. Rev. B 29, 1685 (1984).

55 G. Semenoff, Phys. Rev. Lett. 53, 2449 (1984).
56 N. H. Shon and T. Ando, J. Phys. Soc. Jpn. 67, 2421 (1998).

57 T. Ando, J. Phys. Soc. Jpn. 74, 777 (2005).

58 T. Ando, J. Phys. Soc. Jpn. 38, 989 (1975).

59 S. Latil, V. Meunier, and L. Henrard, Phys. Rev. B 76, 201402 (2007).

60 S. Shallcross, S. Sharma, and O. Pankratov, Phys. Rev. Lett. 101, 56803 (2008).

61 M. Sprinkle, D. Siegel, Y. Hu, J. Hicks, A. Tejeda, A. Taleb-Ibrahimi, P. Le Fèvre, F. Bertran, S. Vizzini, H. Enriquez, et al., Phys. Rev. Lett. 103, 226803 (2009).

${ }^{62}$ P. Moon and M. Koshino, Phys. Rev. B 85, 195458 (2012).

63 T. Ohta, J. Robinson, P. Feibelman, A. Bostwick, E. Rotenberg, and T. Beechem, Phys. Rev. Lett. 109, 186807 (2012). 